\title{
Unexpected EEG Abnormalities in Adults with Parasomnia - A Case Series
}

\author{
Milena K Pavlova*, Saad Yazdani and Ellen J Bubrick
}

Department of Neurology, Harvard Medical School, Boston, Massachusetts, USA

\begin{abstract}
Patients with complex nocturnal behaviors (parasomnia, sleepwalking) can sometimes be difficult to distinguish from those with epilepsy. The differential diagnosis includes identifying abnormalities on electroencephalography (EEG) that are specific for epilepsy - epileptiform discharges. Although these are rarely seen in individuals without epilepsy, and unknown to be associated with sleep disorders, we unexpectedly identified focal epileptiform abnormalities among multiple patients who presented with clinical features suggestive of parasomnia rather than epilepsy-they did not have a history of epileptic seizures or risk factors for epilepsy. The individual history of each patient and the type of abnormality are discussed in the context of any potential for increased cortical irritability.
\end{abstract}

\section{Keywords: Sleep; Parasomnia; Spike; Epilepsy}

\section{Introduction}

Patients with complex nocturnal behaviors (parasomnia, sleepwalking) can sometimes be difficult to distinguish from those with epilepsy. Some types of epileptic syndromes and/or EEG abnormalities only occur in sleep (i.e. Electrical Status Epilepticus of Sleep, Nocturnal Frontal Lobe Epilepsy, some spike-wave discharges seen in a generalized epilepsy), complicating the differential diagnosis further.Helpful steps in the diagnosis include adequate medical history, as well as details about duration, frequency, and character of the behavioral abnormalities. Seizures can be most easily distinguished from parasomnias by distinct abnormalities on electroencephalography (EEG).Specific epileptiform abnormalities can be seen during an epileptic seizure (ictalepileptiform discharges, IED) and in-between seizures - interictalepileptiform discharges (IID). These EEG abnormalities are highly specific for epilepsy and are rarely seen in individuals without history of seizures $[1,2]$.

Generally, an extended EEG montage (a standard 16 channel recording for epilepsy) is recommended to adequately identify epileptiform abnormalities (ICSD). However, many times the differential diagnosis of abnormal nocturnal events remains a challenge for multiple reasons. Patients with some forms of epilepsy, particularly emanating from the frontal lobe, may have infrequent interictal abnormalities [3] which simply may not occur at the time of recording. Frontal lobe epilepsy patients also may have atypical behaviors at night [3]. Lastly, the presence of interical abnormalities, though highly specific for epilepsy, does not by itself prove that the nocturnal behaviors are seizures, as a very small minority of patients may have these abnormalities even in the absence of epilepsy.

In our sleep laboratory, full epilepsy montage is routinely used when nocturnal seizures are suspected as a cause of abnormal nocturnal events, as focal abnormalities may be difficult to identify on the standard six - channel referential montage used for polysomnography (PSG). We were surprised to discover that focal epileptiform abnormalities were seen among multiple patients who presented with clinical features suggestive of parasomnia rather than epilepsy-they did not have a history of epileptic seizures or risk factors for epilepsy. These observations raised the suspicion that IID may be more frequent among patients with parasomnia than among other individuals without epilepsy. We present a series of six patients who presented with typical history of parasomnia yet had abnormal EEG.

\section{Methods}

The patients presented are a subset of a larger analysis on the usefulness of full EEG montage on laboratory PSG recordings. This study was approved by the IRB for analysis of medical records. All patients had attended in-laboratory recordings performed in accordance with the AASM scoring manual. Montage included: EEG, EOG, EMG, EKG, nasal thermistor flow, nasal pressure, pharyngeal snoring, respiratory effort (2 channels), anterior tibialis EMG, $\mathrm{SaO} 2$ and body position. Video-EEG monitoring was performed throughout the recording using anterior temporal, as well as standard 10-20 EEG electrodes. Data were recorded on a Nihon-Kohden system. Automatic spike-seizure detection utilized the Persyst seizure/spike detection software, in addition to visual inspection.

\section{Results}

Of 237 patients referred for combined PSG/EEG studies, 30\% were referred for evaluation of abnormal nocturnal events, while the rest were referred either for evaluation of sleep disorders in patients with known epilepsy or for other indications. Of the patients with no prior known history of epilepsy, $16 \%$ had epileptiform discharges, as described in a prior study from our research team [4]. Below is a description of five patients within this cohort, who presented with parasomnia, were not found to have epilepsy by clinical evaluation, and who had an abnormal nocturnal EEG.

Patient 1: A 22-year-old woman who presented with sleep related eating. The disorder started in young adulthood and significantly worsened after the patient was diagnosed with and treated for bipolar disorder. She never had a childhood history of sleepwalking. Her family reported evidence of eating from the disarray in the kitchen,

*Corresponding author: Milena K. Pavlova, Brigham and Women's -Faulkner Hospital, Department of Neurology, Suite 4970, 1153 Centre St, Boston MA 02130 USA, Tel: 617-983-7580; Fax: 617-983-7582; E-mail: mpavlova1@partners.org

Received August 22, 2014; Accepted November 07, 2014; Published November 12,2014

Citation: Pavlova MK, Yazdani S, Bubrick EJ (2014) Unexpected EEG Abnormalities in Adults with Parasomnia - A Case Series. J Neurol Neurophysiol 5 246. doi:10.4172/2155-9562.1000246

Copyright: (c) 2014 Pavlova MK, et al. This is an open-access article distributed under the terms of the Creative Commons Attribution License, which permits unrestricted use, distribution, and reproduction in any medium, provided the original author and source are credited. 
though she would be amnestic for the events. She reported that the events probably occur in the early part of the night. The patient noted worsening symptoms in the setting of fragmented sleep. Several recent medications included quetiapine, as well as zolpidem for insomnia. There was no report of convulsions, daytime events suggestive of partial seizures, or significant risk factors for epilepsy. Physical examination was within normal limits. Evaluation revealed a single interictal EEG abnormality, displayed in Figure 1, seen on the 6-hour recording. The patient improved after sleep hygiene modification and medication change.

Patient 2: A 36 year old man presented with an injury that was due to abnormal behavior at night (punching a hard object). He reported that events started in adulthood, were highly variable in frequency, and tended to be provoked by a noisy sleep environment. He was amnestic for most events. The timing of the injury suggested that the event occurred in the first third of the night. Types of behaviors included walking out of his bed or bedroom, attempting to switch light on, as well as various arm and leg movements while remaining in bed. There had never been convulsions or any events that could be interpreted as seizures and he was otherwise healthy. Physical exam was unremarkable. Evaluation revealed mild obstructive sleep apnea, as well as a single interictal EEG abnormality, displayed in Figure 2 , on the 6-hour recording. The frequency of the events diminished significantly with improved sleep hygiene, treatment of the sleep apnea, and an intermittent low dose benzodiazepine (taken only at times when he was in a noisy environment).

Patient 3: 44 year old woman with dystonia who also complained of insomnia. She was given Zolpidem for symptomatic treatment and evaluated with a polysomnogram with epilepsy EEG montage to test for co-morbid sleep disorders. At her follow-up visit, she reported that since the start of the Zolpidem treatment she had also started to experience abnormal behaviors during sleep, such as re-arranging objects in her room. She was amnestic for the events, but knew of the behaviors from the changed order of the objects. There were no convulsions, no presence of other events that could be interpreted as epileptiform, and no other medical problems at the time. Polysomnogram revealed IEDs as in Figure 3. Behaviors resolved after the cessation of Zolpidem.

Patient 4: A 25 year old man with complex nocturnal behaviors that started in high school. The patient described variable movements, including talking, laughing, and gesturing usually in the first part of the night. On some occasions, the patient left the bed and sometimes sustained minor injuries. There were no convulsions reported. His past medical history revealed asthma and was not significant for other health problems or medications. Evaluation revealed moderate obstructive sleep apnea, as well as a single interictal abnormality (Figure 4). An event was recorded during the study - it started during slow wave sleep with laughter, followed by sleep talking and subsequently sitting in bed. There were no epileptiform EEG abnormalities during the event. The patient had surgical treatment for OSA and a subsequent standard (6-EEG channel) polysomnography indicated improved breathing. Despite the treatments, the patient continued to have abnormal nocturnal behaviors.

Patient 5: 46 year old woman with history of parasomnia since childhood. The patient's events occurred during the first half of the night. During adulthood, the frequency of the events ranged from 3 times per week to twice per month and consisted of talking, screaming, agitation and movements of the body and extremities. After an event, the patient typically would fall asleep. At times, there was an associated sense of a dream fragment. On rare occasions, she would wake up outside of her bed and appeared to be trying on clothes or taking a shower. There were no associated injuries. In her early years, consideration was given to seizures and she had evaluation with CT and MRI of the brain.These studies were normal by report. VideoEEG evaluation revealed asymmetric theta in the left anterior temporal

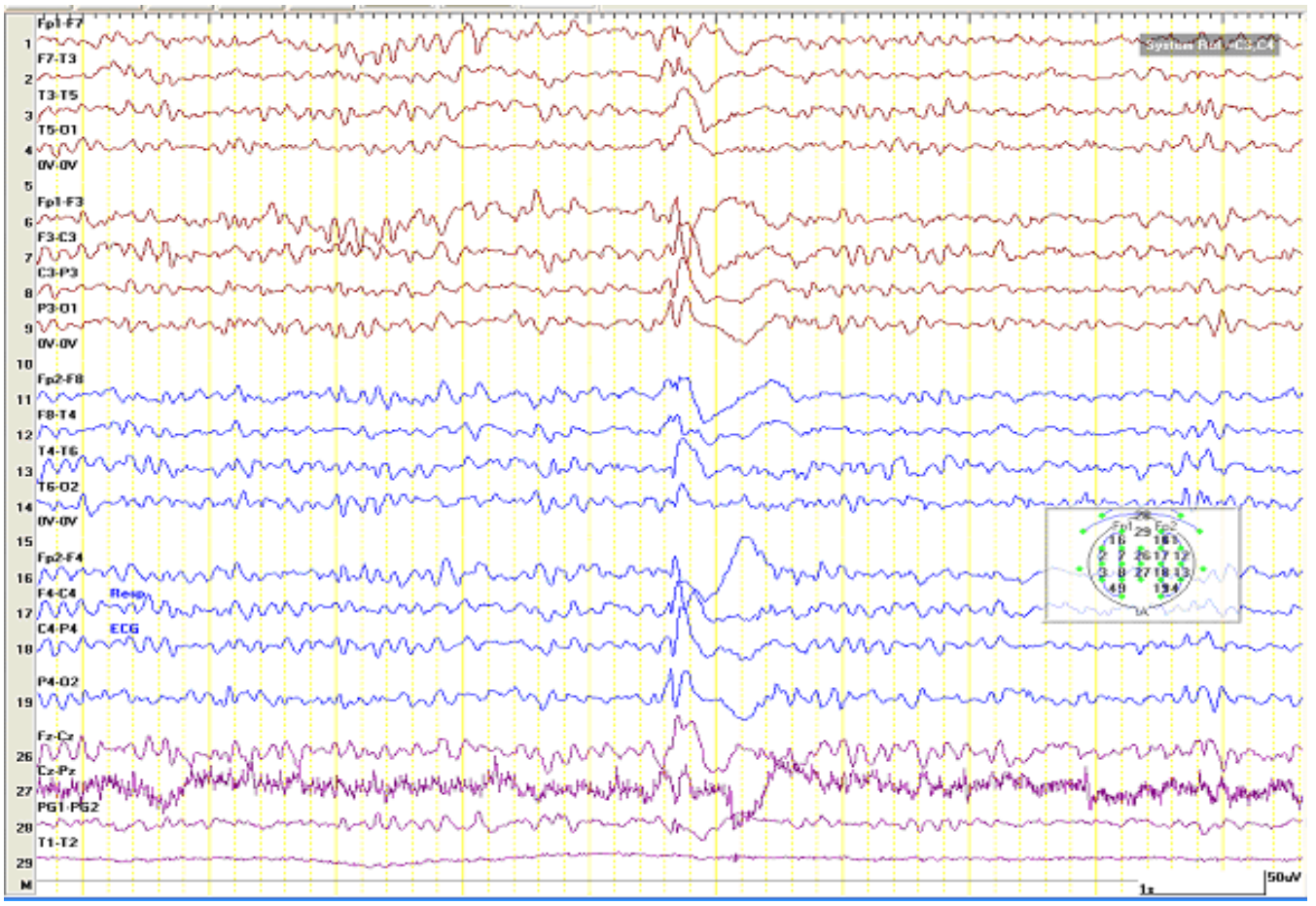

Figure 1: Ten-second page of EEG from patient 1, demonstrating an abnormally shaped $\mathrm{K}$ complex. A sharp component is seen with a phase reversal around P4. 


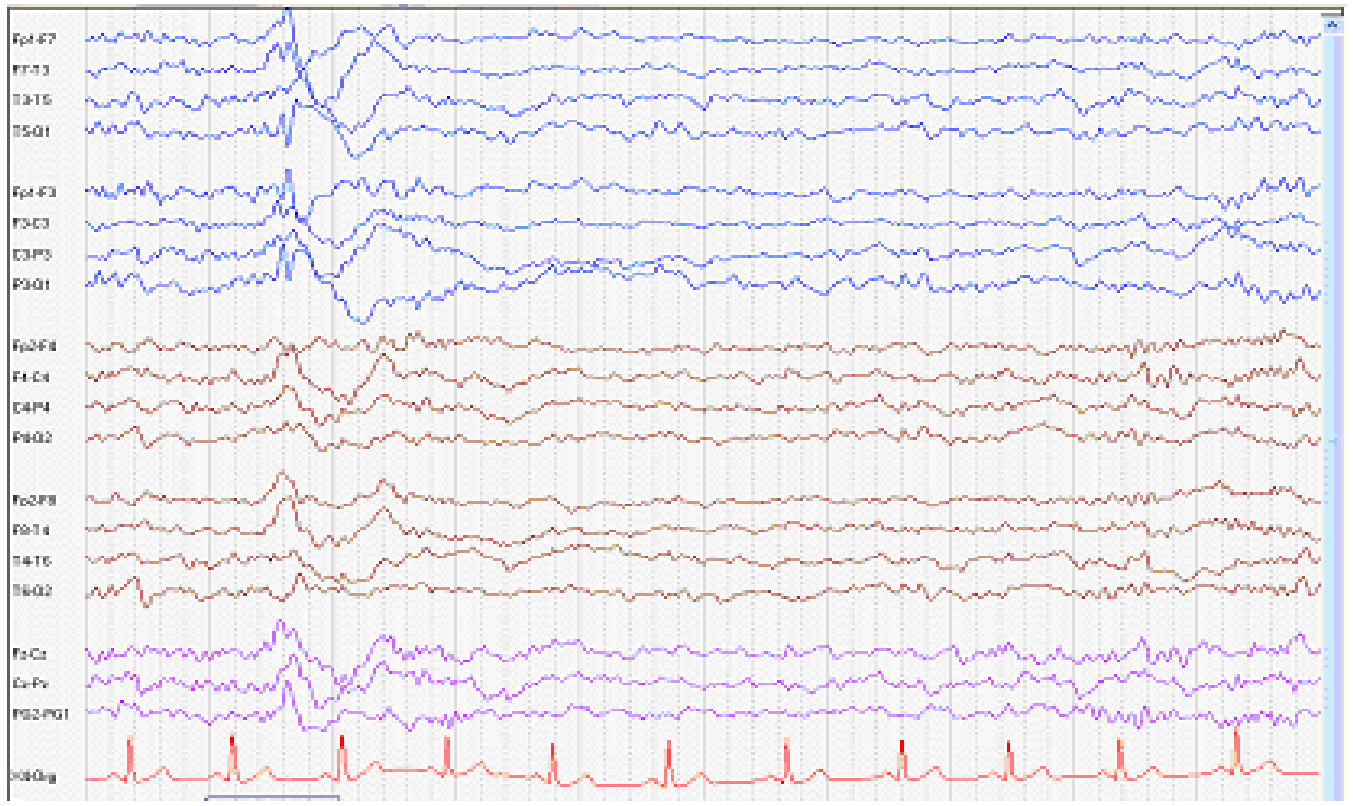

Figure 2: Ten-second page of EEG from patient 2, demonstrating an abnormally shaped $\mathrm{K}$ complex. A sharp component is seen with a broad field and phase reversal around $\mathrm{C} 3$.

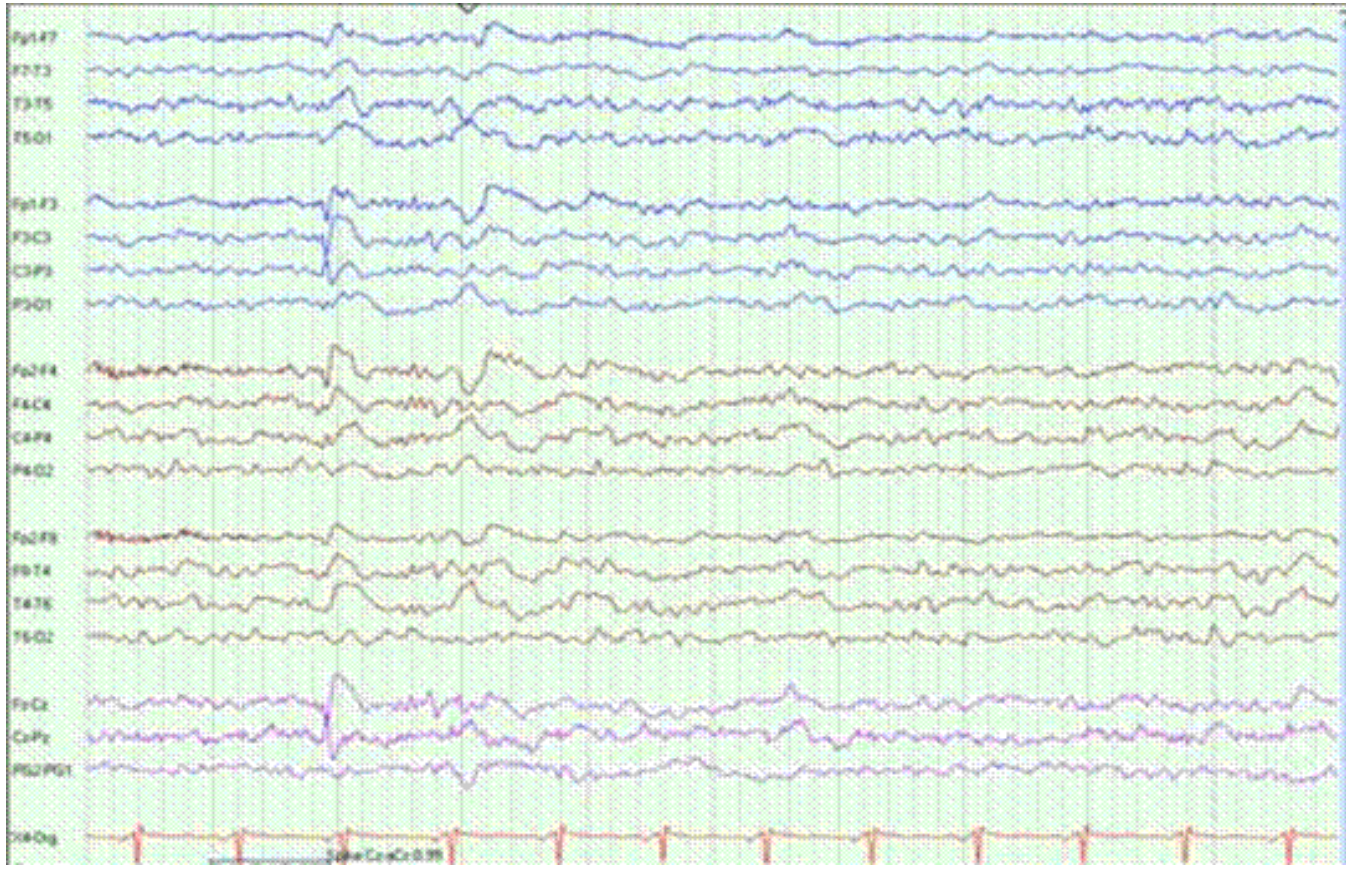

Figure 3: Ten-second page of EEG from patient 3, demonstrating a sharp wave with phasereversal around C3.

region.A representative segment of the overnight EEG is in Figure 5. The patient also had obstructive sleep apnea. The parasomnia events resolved with optimal treatment with CPAP.

\section{Discussion}

We unexpectedly discovered EEG abnormalities, mainly epileptiform in nature, in multiple adults with complex sleep related behaviors in the absence of evidence of forthright epilepsy. Our report is preliminary - it consists of clinically-identified patients - as opposed to prospective, which would provide a more accurate measure of frequency and character of the abnormalities. Interestingly, although EEG abnormalities can be seen rarely in healthy individuals [1], the rate of EEG abnormalities present in our patients appears to be much higher than expected by chance alone.

Multiple studies have evaluated ways to differentiate a seizure from a parasomnia event [5,6,4]. Many of these have included helpful elements in the history, EEG [3,7,5], or have reported sleep instability or abnormalities of a specific sleep stage as a potential neurophysiologic 


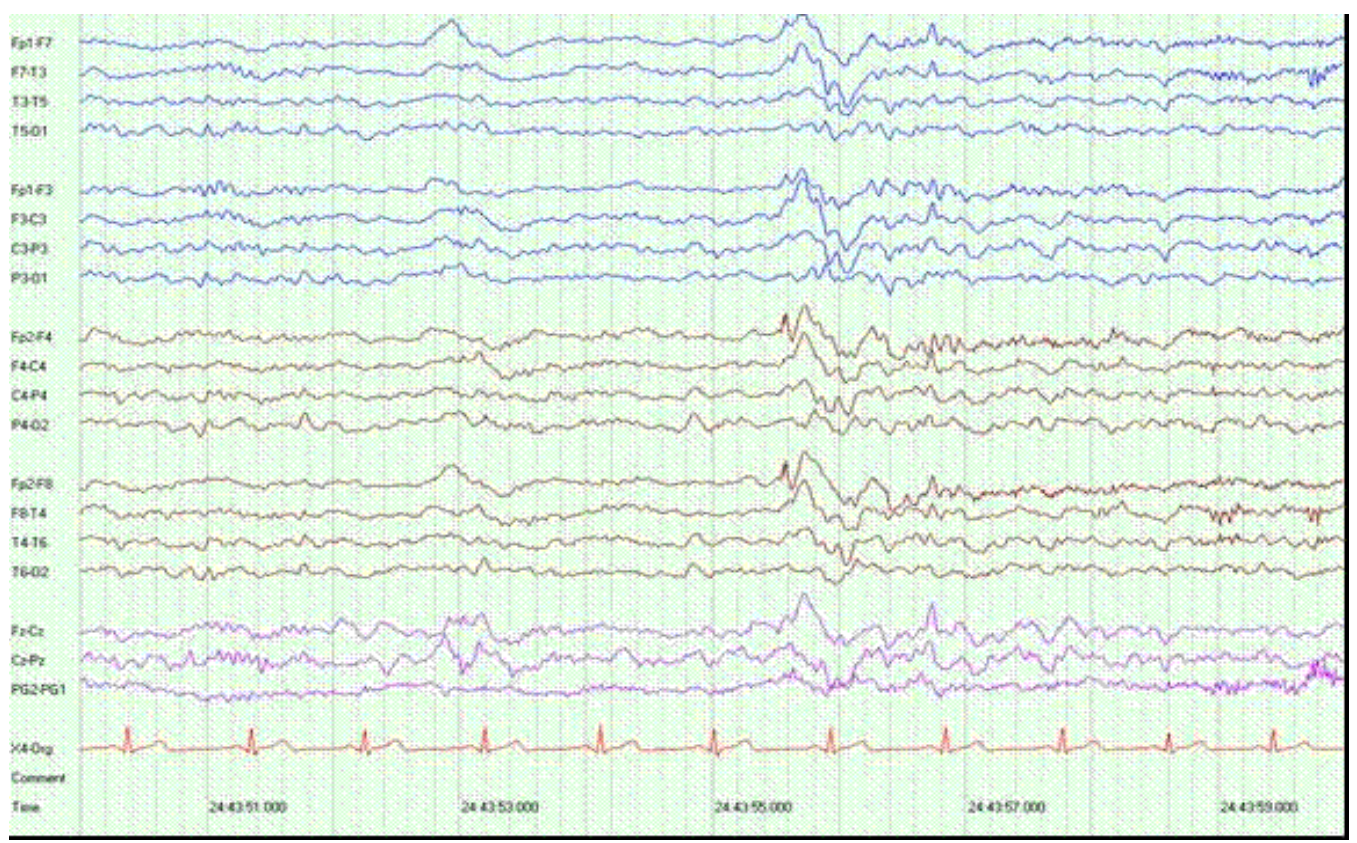

Figure 4: Ten-second page of EEG from patient 5 , demonstrating an abnormally shaped $\mathrm{K}$ complex. On the top panel, a sharp component is seen with a likely frontal location.Bottom panel demonstrates the same abnormality on transverse montage. The phase reversal appears at FP2.

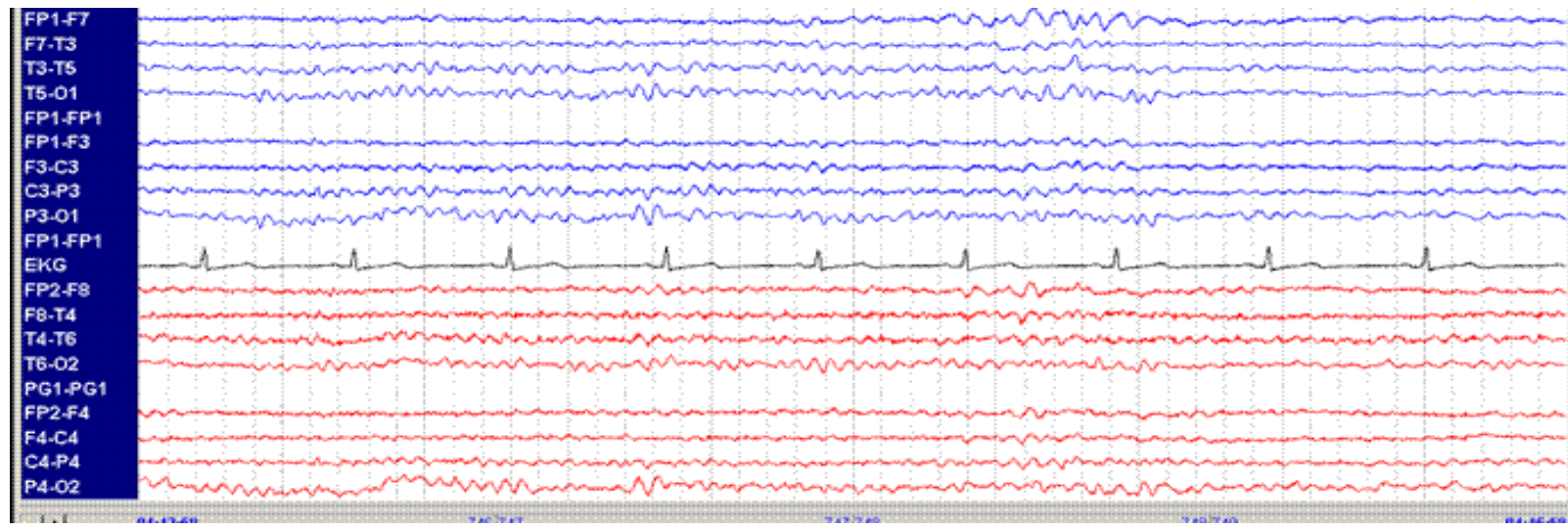

Figure 5: Ten-second page of EEG from patient 4, demonstrating asymmetric rhythmic theta. Maximal negativity appears around F7-T3.

cause of parasomnia [8]. Precious little, however, is reported about EEG abnormalities or neurophysiologic features specific to patients with parasomnia only. Hypersynchronous delta has been reported in association with parasomnia [9], though this is a non-specific finding and not associated with cortical irritability (Table 1).

Parasomnia and epilepsy may co-exist in the same patient. For example, a recent case report described a patient with a ten-year history of epilepsy who subsequently developed catathrenia [10]. Additionally, Silvestri et al described six patients with a history of sleepwalking, but subsequently developed epilepsy [11]. She also reported patients with nocturnal frontal lobe epilepsy who presented with restless legs like symptoms [12]. Similar to the aforementioned studies, it is possible that our patients may have been at a higher risk of epilepsy due to the EEG abnormalities, however none of the five patients developed definite epileptic seizures within the time of follow-up (3 months to 4 years).
Our patients differed in age, gender, comorbidities, and semiology of the events, however all had fairly complex sleep-related behaviors including some form of sleepwalking. Specific features included timing of the events, complexity, lack of clustering, and lack of stereotypy, as well as occurrence from slow wave sleep [3]. Overall, the clinical characteristics of the behaviors strongly favor parasomnia.

In two of the patients, the events appeared to be provoked by zolpidem. Zolpidem treatment has been associated with complex nocturnal behaviors as a possible side effect, but not with seizures or EEG abnormalities $[13,14,15]$. Additionally, four of the patients had no apparent provoking medications and therefore medication use did not appear to be a consistent causative factor.

In three of the five patients, the EEG abnormalities tended to occur in association with a K-complex, causing an abnormal shape of the K-complex itself. K-complexes are normal EEG features that emerge in stage N2 sleep[16]. Historically, during the early years of 


\begin{tabular}{|c|c|c|c|c|c|}
\hline & Patient 1 & Patient 2 & Patient 3 & Patient 4 & Patient 5 \\
\hline Age & 22 & 36 & 44 & 25 & 46 \\
\hline Gender & Female & Male & Female & Male & Female \\
\hline $\begin{array}{l}\text { Approximate age of } \\
\text { onset }\end{array}$ & Adult & Adult & Adult & Late teens & $\begin{array}{l}\text { Childhood, persisted in } \\
\text { adulthood }\end{array}$ \\
\hline $\begin{array}{l}\text { Childhood history of } \\
\text { sleep walking }\end{array}$ & No & No & Yes & None prior & Yes \\
\hline Comorbid sleep disorder & Insomnia & Mild OSA (RDI=10/hr) & Insomnia & OSA & OSA \\
\hline Other comorbidities & Bipolar disorder & None & Dystonia & None & None \\
\hline Medications & $\begin{array}{l}\text { Zolpidem, } \\
\text { quietapine }\end{array}$ & None & Zolpidem & None & None \\
\hline Epilepsy risk factors & $\mathrm{N} / \mathrm{a}$ & None & None & None & None \\
\hline $\begin{array}{l}\text { Known history of } \\
\text { seizures }\end{array}$ & No & No & No & No & No \\
\hline Sleep fragmentation & $\begin{array}{c}\text { Frequent } \\
\text { awakenings }\end{array}$ & Awakenings, due to noise & Yes & Yes & Yes \\
\hline Time of night & First half & Middle & Unknown & Variable & First half \\
\hline Frequency of events & Variable & $\begin{array}{l}\text { Variable - more frequent } \\
\text { when sleep fragmented }\end{array}$ & $\mathrm{N} / \mathrm{a}$ & Almost every night & $\begin{array}{l}\text { Three per week in childhood, two } \\
\text { per month in adulthood }\end{array}$ \\
\hline Main event type & $\begin{array}{l}\text { Sleep eating, } \\
\text { occasional } \\
\text { sleepwalking }\end{array}$ & $\begin{array}{l}\text { Sleepwalking, arm/leg } \\
\text { movements, talking }\end{array}$ & $\begin{array}{l}\text { Sleepwalking, re-arranging } \\
\text { objects, complex behaviors }\end{array}$ & $\begin{array}{l}\text { Sleepwalking, talking, } \\
\text { laughter, gesturing, complex } \\
\text { behaviors }\end{array}$ & $\begin{array}{l}\text { Sleepwalking, talking, } \\
\text { screaming, agitation, complex } \\
\text { behaviors }\end{array}$ \\
\hline Stereotypical events & No & No & No & No & No \\
\hline Oriented after event & Not witnessed & Yes & Not witnessed & Yes & Not witnessed \\
\hline Amnesia & Yes & Yes & Yes & Yes & Yes \\
\hline $\begin{array}{l}\text { Probable provoking } \\
\text { factors }\end{array}$ & $\begin{array}{l}\text { Zolpidem, } \\
\text { fragmented sleep }\end{array}$ & Noise, sleep fragmentation & Zolpidem & None known & OSA \\
\hline Use of Zolpidem & Yes & No & Yes & No & No \\
\hline MRI/other evaluations & $\mathrm{N} / \mathrm{a}$ & Normal MRI & $\mathrm{N} / \mathrm{a}$ & $\mathrm{N} / \mathrm{a}$ & Normal MRI, CT \\
\hline Improvement with & $\begin{array}{l}\text { Corrected sleep } \\
\text { hygiene, stop } \\
\text { Zolpidem }\end{array}$ & Lorazepam, OSA treatment & Stop Zolpidem & $\begin{array}{l}\text { Surgical treatment of OSA; } \\
\text { events mostly persisted }\end{array}$ & $\begin{array}{l}\text { Events resolved with optimal } \\
\text { CPAP use }\end{array}$ \\
\hline
\end{tabular}

Table 1: Clinical characteristics of the five adults.

\begin{tabular}{|c|c|c|c|c|c|}
\hline & Patient 1 & Patient 2 & Patient 3 & Patient 4 & Patient 5 \\
\hline $\begin{array}{l}\text { Frequency of EEG abnormalities (on a } \\
6 \text { hour recording) }\end{array}$ & One & One & One & One & Rare \\
\hline Type of EEG abnormalities & $\begin{array}{l}\text { Single abnormal asymmetric } \\
\text { sharply contoured K complex }\end{array}$ & $\begin{array}{c}\text { Single abnormal } \\
\text { asymmetric sharply } \\
\text { contoured K complex }\end{array}$ & Sharp wave & $\begin{array}{l}\text { Single abnormal asymmetric } \\
\text { sharply contoured } \mathrm{K} \text { complex }\end{array}$ & $\begin{array}{l}\text { Asymmetric focal } \\
\text { rhythmic sharp theta }\end{array}$ \\
\hline Location of EEG abnormalities & P4 (right parietal) & C3 (left central) & C3 (left central) & FP2 (right frontal) & F7-T3 \\
\hline
\end{tabular}

Table 2: EEG abnormalities of the patients.

EEG recording, technicians sometimes provoked them by stimulation, which has been reported to have established their name ( $\mathrm{K}$ - for "knocking"). Occasionally, arousals from sleep may consist of a series of K-complexes [17]. Dyshormia - a paroxysmal response to arousing stimuli, electrographically characterized as an abnormal $\mathrm{K}$ complex associated with a spike has been described by a number of authors $[18,19,20]$. It is thought to reflect an abnormal arousal with cortical irritability. In all instances, however, it was described in association with epilepsy, not parasomnia.

The location and character of the EEG abnormalities differed - left temporal in three, frontal in one, and central in one. However, in all patients the abnormalities were very rare and only one abnormality was seen through the entire 6-7 hour recording. If these patients were evaluated with a routine 20 minute EEG, as is usually performed in clinical practice, it is likely that these rare abnormalities would not be captured. The overnight PSG/full head EEG recording provides a higher sensitivity[4].

Our unexpected findings lead us to suspect that parasomnia may be associated with cortical irritability. Physiologically, we could speculate that the hypersynchrony of cerebral neurons that lead to an epileptiform discharge may be provoked in a similar way as a $\mathrm{K}$ complex,by a minor stimulus that is not sufficient to cause a full arousal, but may trigger a partial EEG response. To our knowledge, this would be a new way of conceptualizing and hence approaching parasomnia patients. Perhaps a more comprehensive evaluation that includes more extended, overnight EEG monitoring should be considered as part of the evaluation. Further studies will be needed to evaluate the prevalence of these abnormalities in the parasomnia population, and their clinical significance.

\section{References}

1. Capdevila OS, Dayyat E, Kheirandish-Gozal L, Gozal D (2008) Prevalence of epileptiform activity in healthy children during sleep. Sleep Med 9: 303-309.

2. Gregory RP, Oates T, Merry RT (1993) Electroencephalogram epileptiform abnormalities in candidates for aircrew training ElectroencephalogrClinNeurophysiol 86: 75-77.

3. Derry CP, Harvey AS, Walker MC, Duncan JS, Berkovic SF (2009) NREM arousal parasomnias and their distinction from nocturnal frontal lobe epilepsy: a video EEG analysis. Sleep 32: 1637-1644

4. Bubrick EJ, Yazdani S, Pavlova MK Beyond standard polysomnography: Advantages and indications for use of extended 10-20 EEG montage during laboratory sleep study evaluations. Seizure.2014 Oct;23(9):699-702. 
Citation: Pavlova MK, Yazdani S, Bubrick EJ (2014) Unexpected EEG Abnormalities in Adults with Parasomnia - A Case Series. J Neurol Neurophysiol 5: 246. doi:10.4172/2155-9562.1000246

Page 6 of 6

5. Foldvary-Schaefer N, Grigg-Damberger M (2009) Sleep and epilepsy. SeminNeurol 29: 419-428.

6. YiÅ̈̈ U, Kurul SH, Ã-ztura I, Ecevit MC, Dirik E (2013) Polysomnographic and long-term video electroencephalographic evaluation of cases presenting with parasomnias. ActaNeurolBelg 113: 285-289.

7. Bisulli F, Vignatelli L, Provini F, Leta C, Lugaresi E, et al. (2011) Parasomnias and nocturnal frontal lobe epilepsy (NFLE): lights and shadows--controversial points in the differential diagnosis. Sleep Med 12 Suppl 2: S27-32.

8. Hughes JR (2007) A review of sleepwalking (somnambulism): the enigma of neurophysiology and polysomnography with differential diagnosis of complex partial seizures. Epilepsy Behav 11: 483-491.

9. Pilon M, Zadra A, Joncas S, Montplaisir J (2006) Hypersynchronous delta waves and somnambulism: brain topography and effect of sleep deprivation. Sleep 29: 77-84.

10. Zinke J, Rupprecht S, Schwab M, Hagemann G (2010) Nocturnal groaning (catathrenia) and epilepsy. Epileptic Disord 12: 136-137.

11. Silvestri $R$, de Domenico $P$, Mento $G$, Laganà A, di Perri R (1995) Epileptic seizures in subjects previously affected by disorders of arousal. NeurophysiolClin 25: 19-27.

12. Aricò I, Condurso R, Granata F, Nobili L, Bruni O, et al. (2011) Nocturnal frontal lobe epilepsy presenting with restless leg syndrome-like symptoms. NeurolSci 32: $313-315$

13. Gibson CE, Caplan JP (2011) Zolpidem-associated parasomnia with serious self-injury: a shot in the dark. Psychosomatics 52: 88-91.

14. Miranda M, Seijas D, Castillo JL, Pérez J C (2010) [Zolpidem induced sleep related eating disorder]. Rev Med Chil 138: 1067-1069.

15. Wing YK, Lam SP, Li SX, Zhang J, Yu MW (2010) Sleep-related eating disorder and zolpidem: an open interventional cohort study. J Clin Psychiatry 71: 653656.

16. Kryger R, and Dement, eds (2010) Principles and Practice of Sleep Medicine.

17. AASM (2007) The AASM Manual for the Scoring of Sleep and Associated Events: Rules, Terminology and Technical Specifications Version 2.0

18. Niedermeyer E (2008) Epileptiform K complexes. Am J ElectroneurodiagnosticTechnol 48: 48-51.

19. Nowack WJ (1996) Dyshormia revisited: generalized seizures and arousal. ClinElectroencephalogr 27: 22-25.

20. Geyer JD, Carney PR, Gilliam F (2006) Focal epileptiform spikes in conjuction with K-complexes. J ClinNeurophysiol 23: 436-439. 